Aims The purpose of this work is to study features of physical development in children with cerebral palsy (CP) brought up in the different conditions.

Methods Sixty children with CP participated in the research. Children were divided into 2 groups: the main group consisted of children brought up in children's community, and the comparison group consisted of children brought up in a family. Physical development of children was assessed using the WHO ANTHRO program, at the same time the body mass index (BMI) and indicators of $\mathrm{Z}$-score $\mathrm{BMI}$ concerning age were calculated.

Results When studying physical development of children it is established that at children of the main group average size BMI was $15.47 \pm 2.65$, and the comparison group - 16.21 \pm 2.89 .

Easy insufficiency of nutrition (Z-score from $-2 \sigma$ to- $1 \sigma$ ) was observed at $26.7 \%$ of the examined children of the main group, and at $23.3 \%$ at the comparison group. Moderate nutritional deficiency (Z-score from $-3 \sigma$ to $-2 \sigma$ ) it was diagnosed for $23.3 \%$ of children of the main group and for $16.7 \%$ at the comparison group, while the largest number of children with moderate nutritional deficiency was determined at the age of 3 to 4 years in both groups. Heavy degree of nutritional deficiency (Z-score $<-3 \sigma$ ) in the compared groups was noted equally (6.7\%).

Overweight corresponding to a moderate increase in nutrition (Z-score from $+1 \sigma$ to $+2 \sigma$ ) was detected in $10 \%$ of children in the main group and in the $6.7 \%$ at the comparison group, increased moderate nutrition (Z-score from $+2 \sigma$ to $+3 \sigma$ ) was determined in $3.3 \%$ of children of the main group and the comparison group.

Studying of correlation dependence between indicators of BMI and weight at the birth did not reveal reliable communication between signs in the studied groups, at the same time the correlation coefficient in the main group was $r=0.205$, and in group comparison of $r=-0.146(p>0.05)$.

Conclusion Assessment of physical development of children with use of the international standards allowed to establish existence of disharmonious development in most of the examined children.

\section{G40(P) A CLINICAL SERVICE EVALUATION OF PSYCHOLOGY PROVISION FOR CHILDREN AND YOUNG PEOPLE WITH EPILEPSY, AND THEIR FAMILIES}

${ }^{1} \mathrm{R}$ Govindan, ${ }^{2} \mathrm{R}$ Ransley, 'IJ Scott-Blagrove, ${ }^{2} \mathrm{D}$ Wood. 'Paediatrics, Hillingdon Hospital, London, UK; ${ }^{2}$ Paediatric psychology, Hillingdon Hospital, London, UK

10.1136/archdischild-2020-rcpch.28

This service evaluation aims to ascertain if there is a need for increased psychology provision for children and young people with a diagnosis of epilepsy.

Sixteen parents completed a survey based on the PedsQL Epilepsy module - Parent version (Young Children-Varni, 1988) and the Strength and Difficulties Questionnaire (Goodman,1997) prior to their child's outpatient epilepsy appointment. The child or young person's level, impact and longevity of difficulties, in addition to the family burden were measured. Each question was scored and presented in a pie chart.

Two parents reported that their child and family had previous psychological support which was helpful. $81 \%$ of parents reported that their child had some level of difficulties impacting their emotions, concentration, behaviour and relationships and $75 \%$ reported some level of concern about their child's quality of life in relation to their epilepsy. $37.5 \%$ of parents felt that these difficulties cause their child quite a lot of distress, and in $12.5 \%$, a great deal of distress. $62.5 \%$ of parents report that these difficulties have been present for over a year. $68.8 \%$ of parents said that their child's difficulties caused some level of burden, with $12.5 \%$ report feeling a great deal of burden on their family. $62.5 \%$ of parents stated that it would be very likely that they would engage with psychological support.

The majority of parents who completed the survey felt that epilepsy impacts negatively on their child's emotions, concentration, behaviour, relationships and quality of life and they would consider engaging with psychological support around their child's epilepsy. The findings emphasise that this population has an increased level of need for support around their psychological wellbeing. Moreover, there is a desire for additional psychological support for children and young people with epilepsy, and their families. Recommendations for the service have been made in light of these findings.

\section{G41(P) ABSTRACT WITHDRAWN}

\section{G42(P) EPIDEMIOLOGY AND OUTCOME OF STATUS EPILEPTICUS IN CHILDREN WITH NEW ILAE DEFINITION}

${ }^{1} \mathrm{C}$ Mitchell, ${ }^{2} \mathrm{~L}$ Dickson, ${ }^{2} \mathrm{~A}$ Ramsay, ${ }^{3} \mathrm{P}$ Leonard, ${ }^{3} \mathrm{C}$ Brand, ${ }^{3} \mathrm{~A}$ McLellan, ${ }^{2,3} \mathrm{~J}$ Shetty. ${ }^{1}$ Newcastle Upon Tyne Hospitals NHS Foundation Trust, Newcastle Upon Tyne, UK; ${ }^{2}$ Faculty of Medicine, University of Edinburgh, Edinburgh, UK; ${ }^{3}$ Royal Hospital for Sick Children Edinburgh, NHS Lothian, Edinburgh, UK

\subsection{6/archdischild-2020-rcpch.29}

Background Status Epilepticus (SE) in children carries significant risk of morbidity and mortality. Previous work has predominantly focused on $\mathrm{SE} \geq 30 \mathrm{~min}$ but a new ILAE definition has been produced following evidence that seizures $\geq 5 \mathrm{~min}$ are associated with negative outcomes. This study aims to evaluate the epidemiology and outcome of SE since the introduction of buccal midazolam, change in ILAE definition and increased involvement of specialist epilepsy nurses.

Methods Multiple datasets were combined to identify all children presenting to accident and emergency $(\mathrm{A}+\mathrm{E})$ between 2011-2017 in the region. Data was collated from electronic health records; including patient demographics, clinical characteristics, acute seizure management and outcomes. This data can be used to study long-term outcomes, including educational outcome, through national data linkage systems.

Results There were 665 children admitted with SE who had 1228 seizure episodes during the study period. SE accounted for $0.38 \%$ (95\%CI $0.34-0.42 \%)$ of annual A+E admissions. Yearly prevalence, calculated using mid-year-population estimate, was 0.8 per 1000 children. $57.3 \%$ of patients were male (95\% CI 53.5-61.1\%) and median age was 3.65 years $(\mathrm{IQR}=6.33, \mathrm{Min}=0.0, \mathrm{Max}=20.97)$. There is a small deprivation effect $(p=0.0006)$ which is most prominent at ages $2-3$. The median number of PS for each child was 1, however, $34.1 \%$ of children had recurrent SE and $5.6 \%$ had $\geq 5$ SE. Median seizure duration was $10 \mathrm{~min} .30 .3 \%$ of seizures lasted between 5-29 min. Recurrent seizures and longer duration 